\title{
Optimization of Perfect Absorbers with Multilayer Structures
}

\author{
Roberto Li Voti ${ }^{1}$
}

Received: 30 October 2017 / Accepted: 22 December 2017

C Springer Science+Business Media, LLC, part of Springer Nature 2018

\begin{abstract}
We study wide-angle and broadband perfect absorbers with compact multilayer structures made of a sequence of ITO and TiN layers deposited onto a silver thick layer. An optimization procedure is introduced for searching the optimal thicknesses of the layers so as to design a perfect broadband absorber from $400 \mathrm{~nm}$ to $750 \mathrm{~nm}$, for a wide range of angles of incidence from $0^{\circ}$ to $50^{\circ}$, for both polarizations and with a low emissivity in the mid-infrared. We eventually compare the performances of several optimal structures that can be very promising for solar thermal energy harvesting and collectors.
\end{abstract}

Keywords Multilayer structure - Optical materials and properties · Perfect absorber · Solar energy collectors · Thermophotovoltaic

\section{Introduction}

The "blackbody," as introduced by Kirchhoff [1], is an ideal body which is a perfect absorber for all incident radiation: this means for all wavelengths, for both polarizations, and for all angles of incidence [2].

Recently, an increasing interest has been shown in the development of nearly perfect absorber materials for applications such as solar energy collectors and more in general for all the thermophotovoltaic applications (TPV), so as to increase the absorbed power for energy harvesting, storage and conversion as well as for the reprocessing of wasted heat in industrial processes [3].

Roberto Li Voti

roberto.livoti@uniroma1.it

1 Dipartimento di Scienze di Base ed Applicate per l'Ingegneria, Sapienza Università di Roma, Via A.Scarpa 16, 00161 Roma, Italy 
A great improvement in the absorption properties of materials has been achieved by using carbon nanotube technology. In 2009 nanomaterials based on vertically aligned single-walled carbon nanotubes exhibiting an absorption of about $98 \%$ of the incoming light in a wide-spectral-range UV-VIS-NIR have been developed [4]. But despite recent advances in the development of carbon nanotubes (CNT) purity assessment tools, the macroscale assessment of the overall surface qualities of commercial CNT materials remains a great challenge, bringing negative impacts on the reliable and consistent nanomanufacturing of CNT products [5,6].

Recently, most of the perfect absorbers are realized with metamaterials where the enhancement of the absorption can be obtained thanks to the excitation of the surface plasmon resonance, for example in gold and silver [7-14]. This can be achieved with structured metallic surfaces [15], microcavities [16], subwavelength hole arrays and opals $[17,18]$. These devices can be designed as efficient solar collectors thanks to the low thermal emissivity of silver and gold.

Alternatively structured phase change materials [19-22] and chiral metamaterials [23-26] have been recently used so as to obtain an active switching of the absorption properties thanks to the metal-insulator transition in one case or to intrinsic/extrinsic dichroism in the other case.

However, the realization of broadband absorber metamaterials requires long and expansive procedures with multiple steps of film deposition, photoresist coating, etching and photoresist removing [27].

Conversely, the design and realization of perfect absorbers with multilayers can be more convenient [28]. The multilayer structure can be easily theoretically tested by numerical simulations and optimized by several search methods (genetic algorithms [29], neural network, steepest descent search method [30,31], etc.) so as to obtain broadband absorbers for a wide range of angles of incidence. In addition, the multilayer optically opaque structures are also easy to be characterized by using many different diagnostic techniques: for example photothermal, photoacoustic and thermographic techniques [32-35].

Concerning the materials, an increasing interest is shown for inorganic ceramic materials such as semiconductor-based oxides and transition metal nitrides (TiN) which represent the alternative plasmonic materials in the visible frequencies [3640], with a good thermal stability and with a low emissivity in the infrared [41,42].

Other common materials for optical electronic device applications and solar cells are the transparent conducting oxides (i.e., ITO) which can support surface plasmon polariton excitations [43-45].

In this paper we study and optimize a multilayer structure based on a stack of ITO/TiN layers deposited onto a thick silver layer so as to design the coating material for a solar thermal collector. Therefore, our purpose is to find the maximum achievable absorbance in the visible, with a low thermal emissivity in the mid-infrared. We performed numerical simulations by changing the number of layers and the layer thicknesses, and we applied the steepest descent search method to find the optimal thicknesses of the multilayer structure. The theoretical results are eventually discussed and compared with other multilayer metallic coatings. 


\section{Design of the Multilayer Coating}

In this section we discuss the procedure to design an optimized ITO/TiN periodic multilayered structure to be used as a coating material for solar thermal collectors. The structure will be designed so as to exhibit the highest average absorbance in the spectral range from $400 \mathrm{~nm}$ to $750 \mathrm{~nm}$ (visible window) for a wide range of incidence angles from $0^{\circ}$ to $50^{\circ}$, but also satisfying the requirement on the thermal emissivity that should be lower than $20 \%$ in the mid-infrared, so as to minimize the radiative losses of the solar collector.

The concept of multiband perfect absorbers by using metallo-/dielectric periodic multilayers has been recently introduced [46]. It is known that such a particular geometry, which regularly alternates metallic and dielectric layers (1D photonic band gap), can be tailored for specific photonic devices to exhibit exceptional properties, for example to obtain the transparency in metals $[47,48]$, or to achieve the total absorbance [46] in a wide range of wavelengths. Regarding the perfect absorbance, tailored metallo/dielectric periodic structures can fulfill three important requirements at the same time, when a large number of layers (periods) are applied:

(i) To act as antireflection coating so as to minimize the sample reflectance in the visible [49,50];

(ii) To maximize the absorbance in the metallic layers;

(iii) To guarantee a low thermal emissivity in the mid-infrared thanks to the metal layers.

Taking the advantage of the absorbance properties already obtained with the metallo-/dielectric periodic structures, we want to show that it is possible to achieve better results by replacing the standard metal layers with titanium nitride (TiN) layers $[39,40]$.

In fact TiN is an inexpensive metal alloy with smaller free carrier concentrations than noble metals. It behaves as a diluted metal but with material losses comparable to that of gold, showing an optical penetration depth of about $20-30 \mathrm{~nm}$, in the visible range (see Fig. 1 obtained from the data in Ref. [51]). In addition, TiN has also good mechanical hardness, high corrosion resistance, low frictional constant, thermodynamic stability, relatively good thermal conductivity $\left(\approx 20 \mathrm{~W} \cdot \mathrm{m}^{-1} \cdot \mathrm{K}^{-1}\right)$, low thermal emissivity $(<0.2)$, and very high melting temperature of $2930^{\circ} \mathrm{C}$, which makes it suitable for applications such as thermal radiation engineering and thermophotovoltaics [52,53].

In our case the TiN layers are alternated with transparent indium tin oxide (ITO) layers so as to design the ITO/TiN multilayer structure with a suitable number $N$ of periods as shown in Fig. 2. ITO is a transparent conductive oxide, widely used in electronic and optical devices, and compatible with TiN during the deposition process. ITO shows a sufficiently large optical penetration depth of about $700-1000 \mathrm{~nm}$ in the visible range (see Fig. 1 [54]) together with a high refractive index (2.1 at $400 \mathrm{~nm}$, and 1.8 at $800 \mathrm{~nm}$ ), high melting point $\left(>1500^{\circ} \mathrm{C}\right.$ ), and relatively low thermal emissivity.

An earlier study has already shown how a multilayer designed with these two materials (ITO/TiN) can reach an average absorbance of $90 \%$ in the visible range and for a wide range of incidence angles by using 7 layers [39]. However, we show here that this scheme can be further optimized by introducing a silica top layer which 
Fig. 1 Penetration depth as a function of wavelength in the visible range for ITO and TiN
Fig. 2 Scheme of the ITO/TiN periodic multilayer structure $(N$ $=3$ periods, 8 layers)
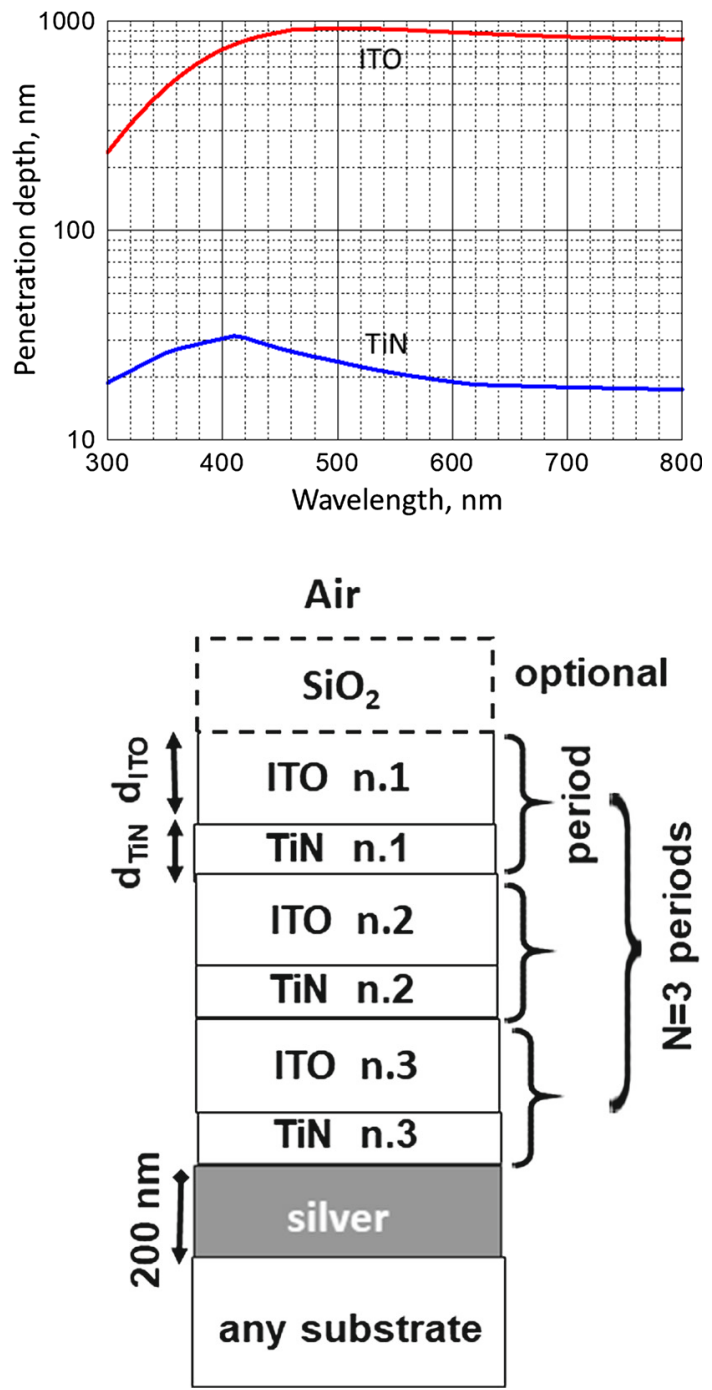

acts as an additional antireflection coating (being $n_{\mathrm{SiO} 2} \cong \sqrt{n_{I T O} \cdot n_{\text {air }}}$ ), without any relevant increase in the thermal emissivity.

The optimal thicknesses of the layers will be found by using the steepest descent search method. This is a first-order iterative optimization algorithm which, in our case, allows to find the local maximum of the average absorbance giving the layer thicknesses with nm precision within 10 iterations.

The optimization of the multilayer structure in Fig. 2 is based on the following approach and constraints:

(a) The structure is made of a sequence of $\mathrm{N}$ periods of identical bilayers ITO/TiN. All the ITO layers have the same thickness $\mathrm{d}_{\text {ITO }}$ chosen in the range $[5-100 \mathrm{~nm}]$ so 
to have layers thinner than $\lambda / 2$ avoiding internal interference effects. All the TiN layers have also the same thickness $\mathrm{d}_{\mathrm{TiN}}$ chosen in the interval [5-80 nm] ranging from transparent to opaque layers. The number of periods $N$ can be selected from 1 to 4 so as to keep the total number of layers $M$ not larger than 10. In fact an increase in the performance is usually expected as the number of periods increases, but for $\mathrm{M}>10$ the performance cannot achieve further substantial improvements, while the realization of the multilayers becomes more difficult, expensive, and critical due to the increasing interfacial effects.

(b) A thin silicon dioxide top layer can be optionally inserted as additional antireflection layer. Its thickness $\mathrm{d}_{\mathrm{SiO} 2}$ can be of $25 \mathrm{~nm}, 50 \mathrm{~nm}$, or $75 \mathrm{~nm}$, so as to increase the absorbance in the visible range, without a relevant change in the IR emissivity. These thicknesses are chosen as multiple of $\lambda / 16$ (at the central wavelength of $600 \mathrm{~nm}$ ) for a fine-tuning of the antireflection condition.

(c) The last $200 \mathrm{~nm}$ thick silver layer absorbs and stops the residual radiation. Therefore, the results of such a numerical simulation are general and independent on the choice of the substrate.

(d) One quantity to be maximized is the absorbance for normal incidence $\mathrm{A}_{\mathrm{o}}$, averaged in the visible range. It is calculated as $A=1-R$ (where $R$ is the reflectance at normal incidence) and integrated in the visible range from $\lambda_{\min }=400 \mathrm{~nm}$ to $\lambda_{\max }=750 \mathrm{~nm}$ as follows

$$
A_{o}=\frac{\int_{\min }^{\lambda_{\max }}(1-R) \mathrm{d} \lambda}{\lambda_{\max }-\lambda_{\min }},
$$

Note that transmittance is neglected due to the thick silver layer.

(e) The final objective is to maximize the absorbance $A_{a v}$ that is averaged twice: in the visible range from $\lambda_{\min }=400 \mathrm{~nm}$ to $\lambda_{\max }=750 \mathrm{~nm}$, and also for a wide range of incidence angles from $0^{\circ}$ to $\theta_{\max }=50^{\circ}$ as follows

$$
A_{a v}=\frac{\int_{0}^{\theta_{\max }} \int_{\lambda_{\min }}^{\lambda_{\max }}(1-R(\theta)) d \lambda d \theta}{\left(\lambda_{\max }-\lambda_{\min }\right) \cdot \theta_{\max }},
$$

where $R(\theta)$ is the reflectance for unpolarized light at the incident angle $\theta$.

(f) The thermal emissivity of the whole structure is also calculated as $\varepsilon_{I R}=1-R_{I R}$ in the infrared range and averaged over the Planck blackbody radiation spectrum at $100^{\circ} \mathrm{C}$. The thermal emissivity should be kept as small as possible $(<0.2)$ to minimize the radiation losses. The calculation is done by using the literature values for the infrared properties of TiN, ITO, $\mathrm{SiO}_{2}$, and silver [51-54]. 


\section{Numerical Simulations}

The simulations of the light propagation in the ITO/TiN multilayer structure have been performed by using the transfer matrix method [55]. According to this method the incoming (outgoing) electric and magnetic fields are contained in the $2 \times 1$ vector $\mathbf{v}_{\mathrm{i}}\left(\mathbf{v}_{\mathrm{o}}\right)$. These two vectors are linked by the linear relationship $\mathbf{v}_{\mathrm{o}}=\mathbf{C}_{\text {tot }} \cdot \mathbf{v}_{\mathrm{i}}$, where $\mathbf{C}_{\text {tot }}$ is the $2 \times 2$ transfer matrix for the whole multilayer, simply given by the product $\mathbf{C}_{\text {tot }}=\mathbf{C}_{\mathrm{M}} \cdot \mathbf{C}_{\mathrm{M}-1} \cdot \mathbf{C}_{\mathrm{M}-2} \cdot \ldots . \mathbf{C}_{2} \cdot \mathbf{C}_{1}$, where $\mathbf{C}_{\mathrm{i}}$ is the $2 \times 2$ transfer matrix of the ith layer as reported in Ref. [55]. By imposing the boundary conditions at infinity, it is possible to calculate reflection and transmission coefficients for the fields, and reflectance and transmittance for the power (in our case transmittance is negligible).

As described in the previous section, the simulations are done for structures made of $N$ periodic repetitions of the fundamental ITO/TiN bilayer with thicknesses $\left[\mathrm{d}_{\mathrm{ITO}} ; \mathrm{d}_{\text {TiN }}\right]$. The quantities $\mathrm{A}_{\mathrm{O}}, \mathrm{A}_{\mathrm{av}}$, and $\varepsilon_{\mathrm{IR}}$ have been calculated as a function of all possible couples of thickness $\left[\mathrm{d}_{\mathrm{ITO}}\right.$; $\left.\mathrm{d}_{\mathrm{TiN}}\right]$ (see Sect. 2), for $N=1,2,3,4$, and without or with a silica top layer $\mathrm{d}_{\mathrm{SiO} 2}$ of $25 \mathrm{~nm}, 50 \mathrm{~nm}, 75 \mathrm{~nm}$.

The optimization procedure follows this approach: first the quantities $N$ and $\mathrm{d}_{\mathrm{SiO} 2}$ are selected, and then, the steepest descent search method is used to find the best couples $\left[\mathrm{d}_{\mathrm{ITO}} ; \mathrm{d}_{\text {TiN }}\right]$ which maximize the quantities $\mathrm{A}_{\mathrm{o}}$ and $\mathrm{A}_{\mathrm{av}}$. Tables 1 and 2 summarize the results coming from such a procedure for optimizing the absorbance $A_{o}$ and $\mathrm{A}_{\mathrm{av}}$, respectively.

Looking at the tables it is worth noting that:

(a) the results in Table 1 slightly differ from the ones in Table 2. The maximum of $\mathrm{A}_{\mathrm{o}}$ (Table 1, col.5) is reasonably a bit larger than $\mathrm{A}_{\mathrm{av}}$ (Table 2, col.5), while the optimal couples $\left[\mathrm{d}_{\mathrm{ITO}} ; \mathrm{d}_{\mathrm{TiN}}\right]$ in cols.3, 4 are practically unchanged from Tables 1 to 2 .

(b) the calculated emissivity for all the structures is very low, fulfilling the requirements (col.6).

(c) for both tables the maxima for $\mathrm{A}_{\mathrm{o}}$ and $\mathrm{A}_{\mathrm{av}}$ are achieved by increasing the number of periods.

(d) For $N=4$, the best performances are obtained by inserting a $50 \mathrm{~nm}$ top layer of $\mathrm{SiO}_{2}$. This layer allows to increase $\mathrm{A}_{0}$ from $96.0 \%$ to $97.0 \%$, and $\mathrm{A}_{\mathrm{av}}$ from $95.4 \%$ to $96.7 \%$ (the data are highlighted in bold), without any relevant change in the emissivity (col.6).

To verify how critical is the choice of the couple $\left[\mathrm{d}_{\mathrm{ITO}} ; \mathrm{d}_{\mathrm{TiN}}\right]$, Fig. 3 shows the contour plot for the quantities $\mathrm{A}_{\mathrm{o}}$ (Fig. 3a) and $\mathrm{A}_{\mathrm{av}}$ (Fig. 3b) as a function of the thicknesses of ITO and TiN layers, without any silica layer, for $N=4$ periods. In Fig. 3 the large area inside the green circles individuates a class of possible structures which guarantees an average absorbance of more than $95 \%$. The maximum of Fig. 3a is shown by the black point at $[47 \mathrm{~nm} ; 16 \mathrm{~nm}]$, while the maximum of Fig. $3 \mathrm{~b}$ is found by the black point at $[49 \mathrm{~nm} ; 16 \mathrm{~nm}]$.

The same procedure can be repeated but with a silica top layer of $50 \mathrm{~nm}$ again for $N=4$ (this is the best case). Figure 4 shows the two contour plots for the quantities $\mathrm{A}_{\mathrm{o}}$ (Fig. 4a) and $\mathrm{A}_{\mathrm{av}}$ (Fig. 4b) to be compared with the analogous contours in Fig. 3. 
Table 1 Results of the procedure to optimize the average absorbance for normal incidence $\mathrm{A}_{\mathrm{O}}$

\begin{tabular}{llllll}
\hline Periods $\mathrm{N}$ & $\begin{array}{l}\text { Thickness } \\
\mathrm{d}_{\mathrm{SiO} 2}[\mathrm{~nm}]\end{array}$ & $\begin{array}{l}\text { Thickness } \\
\mathrm{d}_{\text {ITO }}[\mathrm{nm}]\end{array}$ & $\begin{array}{l}\text { Thickness } \\
\mathrm{d}_{\text {TiN }}[\mathrm{nm}]\end{array}$ & $\mathrm{A}_{\mathrm{O}}[\%]$ & $\varepsilon_{\mathrm{IR}} 100^{\circ} \mathrm{C}$ \\
\hline 1 & None & 46 & 81 & 82.7 & 0.047 \\
1 & 25 & 36 & 81 & 82.4 & 0.044 \\
1 & 50 & 27 & 78 & 80.4 & 0.043 \\
1 & 75 & 25 & 75 & 75.4 & 0.043 \\
2 & None & 51 & 24 & 94.5 & 0.062 \\
2 & 25 & 43 & 24 & 94.0 & 0.059 \\
2 & 50 & 39 & 24 & 91.9 & 0.058 \\
2 & 75 & 47 & 22 & 88.4 & 0.065 \\
3 & None & 54 & 20 & 95.9 & 0.069 \\
3 & 25 & 37 & 16 & 96.2 & 0.065 \\
3 & 50 & 34 & 15 & 95.7 & 0.065 \\
3 & 75 & 43 & 14 & 93.6 & 0.073 \\
$\mathbf{4}$ & None & $\mathbf{4 7}$ & $\mathbf{1 6}$ & $\mathbf{9 6 . 0}$ & $\mathbf{0 . 0 7 0}$ \\
4 & 25 & 41 & 14 & 96.7 & 0.071 \\
$\mathbf{4}$ & $\mathbf{5 0}$ & $\mathbf{2 9}$ & $\mathbf{1 2}$ & $\mathbf{9 7 . 0}$ & $\mathbf{0 . 0 7 1}$ \\
4 & 75 & 34 & 11 & 95.3 & 0.073 \\
\hline
\end{tabular}

Col.1: number of periods $N$; col.2: thickness of the silica layer; col.3 optimized thickness of the ITO layers; col.4: optimized thickness of the TiN layers; col.5: average absorbance $\mathrm{A}_{\mathrm{o}}$, col.6: emissivity calculated as $\varepsilon_{\mathrm{IR}}=1-\mathrm{R}_{\mathrm{IR}}$ and averaged over the Plank blackbody spectrum at $100^{\circ} \mathrm{C}$

Even in this case, a large area exists where the average absorbance is above $96 \%$. The maximum of Fig. $4 \mathrm{a}$ is reached for the couple [ $29 \mathrm{~nm} ; 12 \mathrm{~nm}$ ], while the maximum of Fig. $4 \mathrm{~b}$ is reached for the couple $[30 \mathrm{~nm} ; 12 \mathrm{~nm}]$. From the comparison among Figs. 3 and 4 one observes that the silica top layer of $50 \mathrm{~nm}$ indeed allows to increase the absorbance from $95.4 \%$ to $96.7 \%$. In addition the optimal thickness $\mathrm{d}_{\text {ITO }}$ reduces from $49 \mathrm{~nm}$ to $30 \mathrm{~nm}$ (the ITO and $\mathrm{SiO}_{2}$ layers create a kind of effective $\lambda / 4$ antireflection coating).

In Fig. 4b it is also shown the results of the iterative process during the search of the local maximum of $\mathrm{A}_{\mathrm{av}}$ by using the steepest descent algorithm in the $\left[\mathrm{d}_{\mathrm{ITO}} ; \mathrm{d}_{\text {TiN }}\right.$ ] space. Starting from the initial guess [5.0 nm; $5.0 \mathrm{~nm}$ ] (out of Fig. 4b), the algorithm finds the next couple (small points in Fig. $4 \mathrm{~b}$ ) pointing in the direction of the maximum slope according to the Barzilai-Borwein method. Afterward, it finds [7.8 nm; $8.0 \mathrm{~nm}$ ] at the second iteration, $[10.3 \mathrm{~nm} ; 9.3 \mathrm{~nm}]$ at the third iteration, $[14.1 \mathrm{~nm} ; 10.0 \mathrm{~nm}]$ at the fourth iteration, [20.5 nm; $10.2 \mathrm{~nm}$ ] at the fifth iteration, [26.7 nm; $11.4 \mathrm{~nm}$ ] at the sixth iteration, and giving finally $[29.7 \mathrm{~nm} ; 11.8 \mathrm{~nm}]$ (big point in Fig. 4b) that is rounded with $\mathrm{nm}$ precision in Table 2 . Further iterations to reach a better approximation are useless for practical scopes. Note also that in this case the steepest descent seems the most appropriate search method; in fact it is very easy to be implemented and has a quick convergence because there is only one maximum in the research space. 
Table 2 Results of the procedure to optimize the absorbance $\mathrm{A}_{\mathrm{av}}$ averaged in the visible range and in the range $0^{\circ}-50^{\circ}$

\begin{tabular}{llllll}
\hline Periods N & $\begin{array}{l}\text { Thickness } \\
\mathrm{d}_{\text {SiO2 }[\mathrm{nm}]}\end{array}$ & $\begin{array}{l}\text { Thickness } \\
\mathrm{d}_{\text {ITO }}[\mathrm{nm}]\end{array}$ & $\begin{array}{l}\text { Thickness } \\
\mathrm{d}_{\text {TiN }}[\mathrm{nm}]\end{array}$ & $\mathrm{A}_{\mathrm{av}}[\%]$ & $\varepsilon_{\mathrm{IR}} 100^{\circ} \mathrm{C}$ \\
\hline 1 & None & 48 & 82 & 81.4 & 0.048 \\
1 & 25 & 38 & 82 & 82.0 & 0.044 \\
1 & 50 & 30 & 80 & 80.5 & 0.043 \\
1 & 75 & 26 & 76 & 76.3 & 0.043 \\
2 & None & 53 & 24 & 94.0 & 0.062 \\
2 & 25 & 45 & 24 & 93.6 & 0.059 \\
2 & 50 & 39 & 24 & 91.9 & 0.058 \\
2 & 75 & 47 & 22 & 88.8 & 0.065 \\
3 & None & 58 & 20 & 95.3 & 0.069 \\
3 & 25 & 39 & 16 & 95.7 & 0.066 \\
3 & 50 & 35 & 15 & 95.6 & 0.065 \\
3 & 75 & 43 & 14 & 93.8 & 0.073 \\
$\mathbf{4}$ & None & $\mathbf{4 9}$ & $\mathbf{1 6}$ & $\mathbf{9 5 . 4}$ & $\mathbf{0 . 0 7 0}$ \\
4 & 25 & 41 & 14 & 96.2 & 0.071 \\
$\mathbf{4}$ & $\mathbf{5 0}$ & $\mathbf{3 0}$ & $\mathbf{1 2}$ & $\mathbf{9 6 . 7}$ & $\mathbf{0 . 0 7 1}$ \\
4 & 75 & 34 & 11 & 95.5 & 0.073 \\
\hline
\end{tabular}

Col.1: number of periods $N$; col.2: thickness of the silica layer; col.3 optimized thickness of the ITO layers; col.4: optimized thickness of the TiN layers; col.5: average absorbance $\mathrm{A}_{\mathrm{av}}$, col.6: emissivity calculated as $\varepsilon_{\mathrm{IR}}=1-\mathrm{R}_{\mathrm{IR}}$ and averaged over the Plank blackbody spectrum at $100^{\circ} \mathrm{C}$

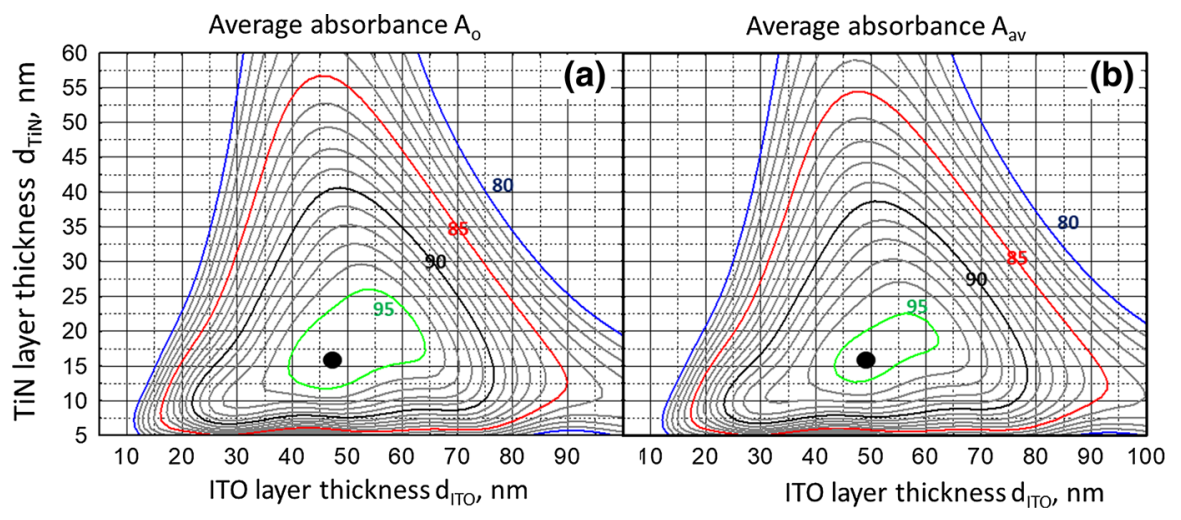

Fig. 3 Contour plot for the quantities $\mathrm{A}_{\mathrm{o}}$ (a) and $\mathrm{A}_{\mathrm{av}}$ (b) as a function of the thickness of the ITO layers $\mathrm{d}_{\text {ITO }}$ and the thickness of the TiN layers $\mathrm{d}_{\mathrm{TiN}} . N=4$ periods. Silica layer is not included

The absorbance spectra at normal incidence is shown in Fig. 5 for the four optimized structures highlighted by the black points in Figs. $3 \mathrm{a}, \mathrm{b}$ and $4 \mathrm{a}$, b. In particular curves (1) and (3) correspond to the optimization of $A_{o}$ and $A_{a v}$ without silica, while curves (2) and (4) correspond to the optimization of $\mathrm{A}_{\mathrm{o}}$ and $\mathrm{A}_{\mathrm{av}}$ when $\mathrm{d}_{\mathrm{SiO} 2}=50 \mathrm{~nm}$ is introduced. 


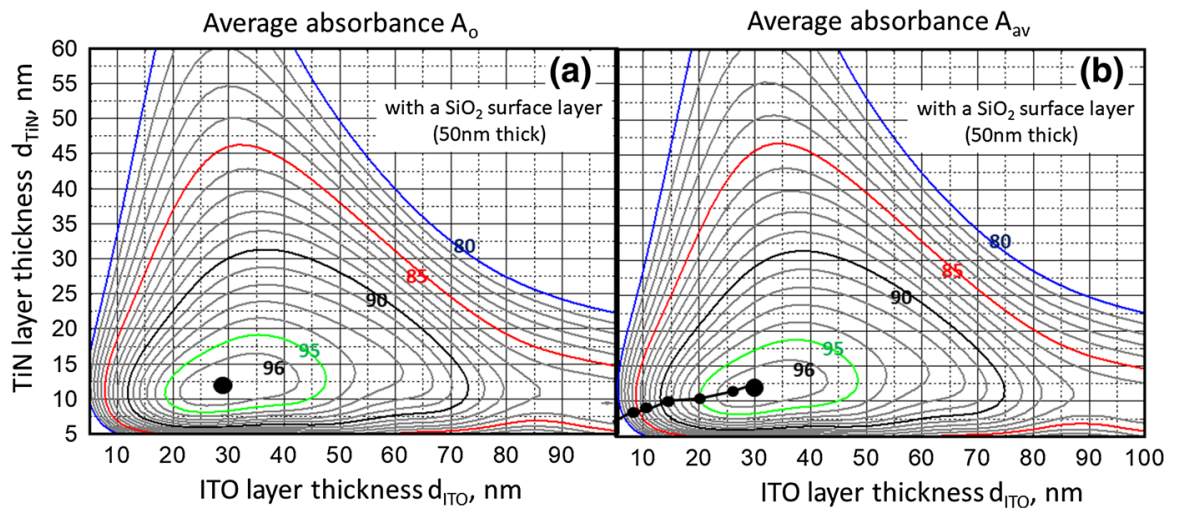

Fig. 4 Contour plot for the quantities $\mathrm{A}_{\mathrm{O}}$ (a) and $\mathrm{A}_{\mathrm{av}}$ (b) as a function of the thickness of the ITO layers $\mathrm{d}_{\text {ITO }}$ and the thickness of the TiN layers $\mathrm{d}_{\mathrm{TiN}} . N=4$ periods. Silica top layer of $50 \mathrm{~nm}$ is included

Fig. 5 Absorbance vs wavelength at normal incidence. The curves refer to the optimized multilayered structures for $N=4$. (1) $\mathrm{A}_{\mathrm{o}}$ is optimized for [47 nm; $16 \mathrm{~nm}$ ] without silica; (2) $A_{o}$ is optimized for $[29 \mathrm{~nm}$; $12 \mathrm{~nm}$ ] with $\mathrm{d}_{\mathrm{SiO} 2}=50 \mathrm{~nm}$; (3) $\mathrm{A}_{\mathrm{av}}$ is optimized for $[49 \mathrm{~nm}$; $16 \mathrm{~nm}$ ] without silica; (4) $A_{a v}$ is optimized for [30 nm; 12nm] with $\mathrm{d}_{\mathrm{SiO} 2}=50 \mathrm{~nm}$

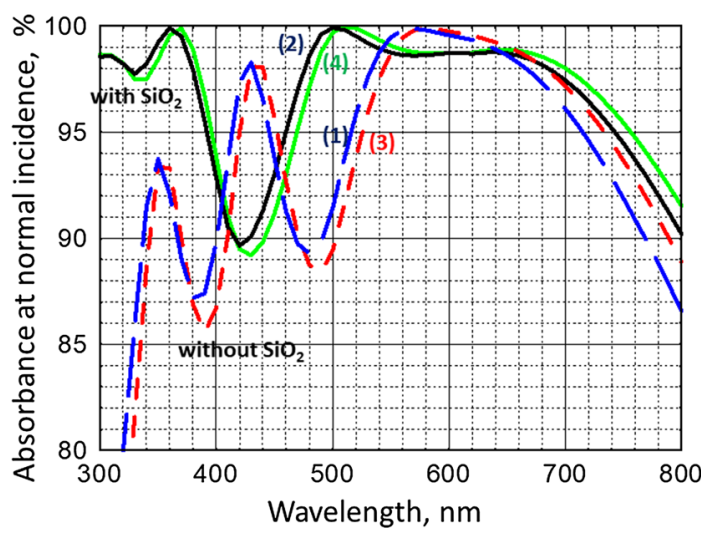

By comparing all curves, the four structures all exhibit a high absorbance in the visible range: no relevant differences are found between curves (1) and (3) and between curves (2) and (4) because the two procedures to optimize $A_{o}$ and $A_{a v}$ found very similar structures. As expected the best absorbance is for the multilayers with $\mathrm{d}_{\mathrm{SiO} 2}=$ 50nm (curves 2,4).

To better understand the absorbing properties of the optimized multilayers, it is useful to study the internal electric field $E$ in the whole structure. Figure 6 shows the amplitude of the E field in each layer for both the $A_{a v}$ optimized structures [49 nm; $16 \mathrm{~nm}$ ] without silica (Fig. 6a) and [30 nm; $12 \mathrm{~nm}$ ] with silica (Fig. 6b) at normal incidence. For a better clarity in abscissa the scale of thickness is amplified so as to have equally spaced layers. Looking at Fig. 6 an efficient mechanism of distributed absorption is seen at the two wavelengths of $600 \mathrm{~nm}$ and $700 \mathrm{~nm}$. Light penetrates without relevant reflection through the structures, being absorbed by the TiN layers and eventually by the silver layer. Conversely the absorption mechanism at $500 \mathrm{~nm}$ is related to the light trapping and quasi-resonant absorption that is enhanced in Fig. $6 \mathrm{~b}$ thanks to the $50 \mathrm{~nm}$ silica top layer [56]. 

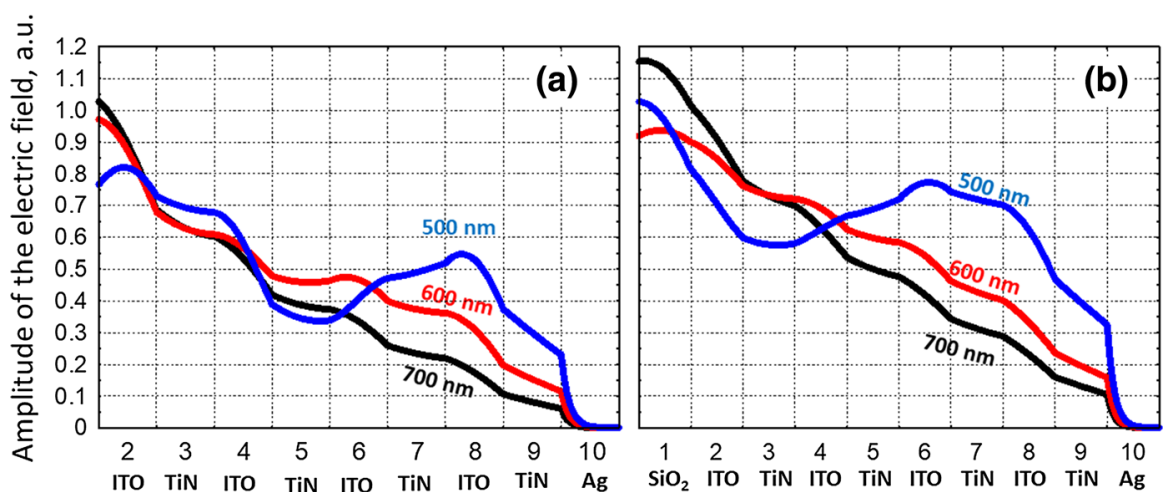

Fig. 6 Amplitude of the internal electric field: (a) $A_{a v}$ optimized structure [ $49 \mathrm{~nm} ; 16 \mathrm{~nm}$ ] without silica; (b) $\mathrm{A}_{\mathrm{av}}$ optimized structure $[30 \mathrm{~nm} ; 12 \mathrm{~nm}]$ with $\mathrm{d}_{\mathrm{SiO} 2}=50 \mathrm{~nm}$. The curves refer to different wavelengths $(500 \mathrm{~nm}, 600 \mathrm{~nm}, 700 \mathrm{~nm})$ for normal incidence. In abscissa the scale of thickness is amplified so as to have equally spaced layers

The different mechanisms of the internal absorption can be put into evidence by calculating the specific absorbance in each layer. Figure 7 shows the distribution of the absorbance for the optimized structure of Fig. 6b ([30 nm; $12 \mathrm{~nm}], N=4$, with $\mathrm{d}_{\mathrm{SiO} 2}=50 \mathrm{~nm}$ ) for normal incidence, at $\lambda=500 \mathrm{~nm}$ (Fig. 7a) and at $\lambda=700 \mathrm{~nm}$ (Fig. 7b). The absorption is mainly concentrated in the TiN layers, but with some differences: at $700 \mathrm{~nm}$ the light is absorbed in the first TiN layers, while at $500 \mathrm{~nm}$, in the central TiN layers due to a resonance process.

The angular absorption properties of the two optimized structures are shown in Fig. 8 at the reference wavelength of $500 \mathrm{~nm}$. The higher absorbance is clearly found for the multilayer where the $50 \mathrm{~nm}$ silica top layer is included (black curves in Fig. 8) for both s, p polarizations, and for a wide range of angles.

To study the analysis on the absorbing properties of the multilayers, the contour plots in Fig. 9 represent a useful tool to put together both the wavelength and angular behavior of the absorbance for the multilayer without silica (Fig. 9a) and with a $50 \mathrm{~nm}$ silica top layer (Fig. 9b). By comparing the two plots the optimized structure with silica shows the best performances in the dotted rectangular area for angles in the range $\left[0^{\circ}-50^{\circ}\right]$ and for wavelengths in the visible range [400-750 nm]. In general the area guarantees an absorbance larger than $98 \%$.

\section{Discussion and Conclusions}

In the previous sections, the approach to design quasi-perfect absorbers based on ITO/TiN periodic multilayered structures has been introduced and deeply discussed. The practical purpose is to find new optimized coatings for solar thermal collectors with the highest achievable absorbance in the visible range from $400 \mathrm{~nm}$ to $750 \mathrm{~nm}$, working for a wide range of angles of incidence from $0^{\circ}$ to $50^{\circ}$, for both polarizations, but with a low thermal emissivity in the mid-infrared, so as to minimize the radiative losses. The results of the optimization procedure show that the performances of the 
Fig. 7 Specific absorbance in each layer $(\%)$ for the optimized structure [30 nm; $12 \mathrm{~nm}$ ], for $\mathrm{N}=4$, with $\mathrm{d}_{\mathrm{SiO} 2}=50 \mathrm{~nm}$ : (a) Normal incidence at wavelength $\lambda=500 \mathrm{~nm}$; (b) normal incidence at wavelength $\lambda=700 \mathrm{~nm}$

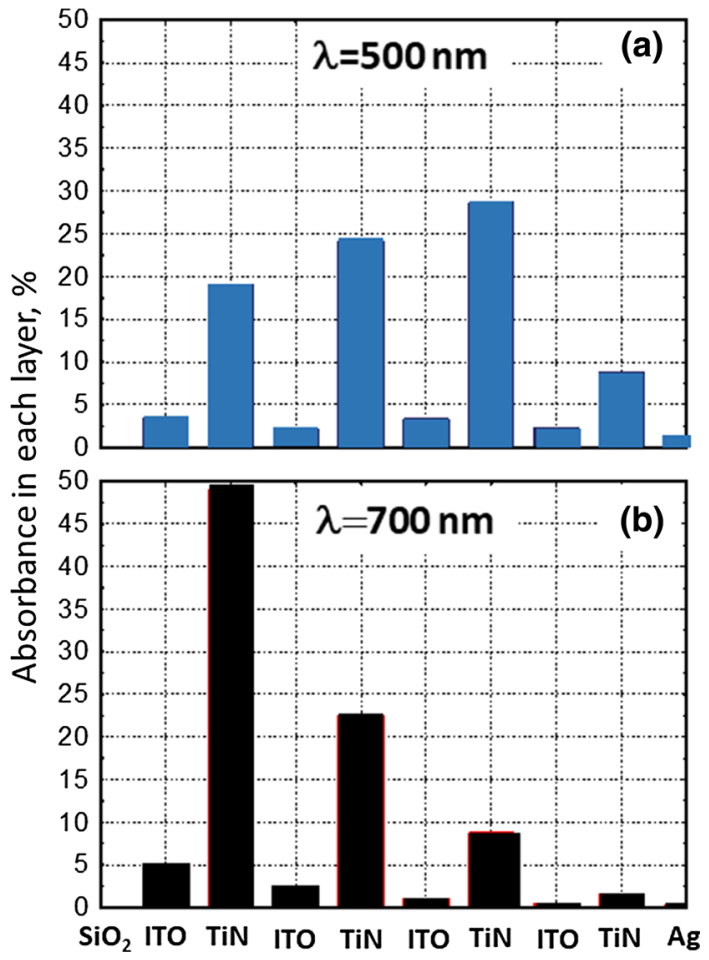

Fig. 8 Absorbance at the reference wavelength of $500 \mathrm{~nm}$ vs angle of incidence (red curves). The $\mathrm{A}_{\mathrm{av}}$ optimized structure [49 nm; $16 \mathrm{~nm}$ ] without silica; (black curves) $\mathrm{A}_{\mathrm{av}}$ optimized structure $[30 \mathrm{~nm}$; $12 \mathrm{~nm}$ ] with $\mathrm{d}_{\mathrm{SiO} 2}=50 \mathrm{~nm}$. Continuous lines are for s-polarized light, dotted lines are for p-polarized light (Color figure online)

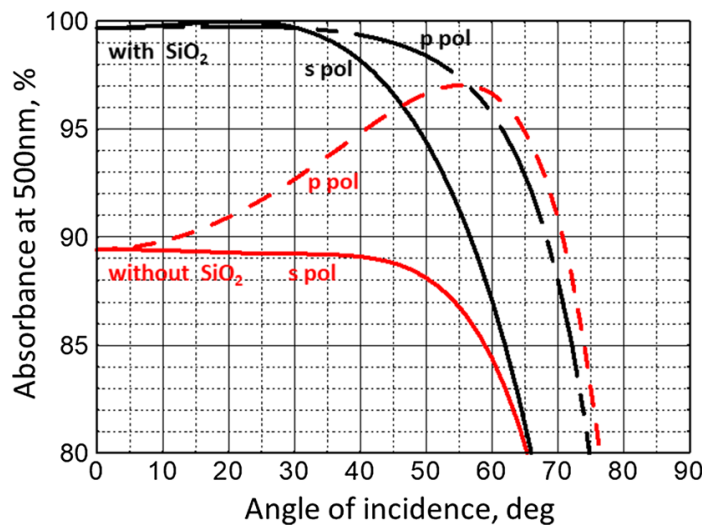

structure increase with the number of periods (layers). For $N=4$ periodical repetitions of the ITO/TiN bilayer [ $49 \mathrm{~nm} ; 16 \mathrm{~nm}$ ], the average absorbance $\mathrm{A}_{\mathrm{av}}=95.4 \%$ is found that can be further improved to $96.7 \%$ by introducing a silica top layer of $50 \mathrm{~nm}$ to the ITO/TiN bilayer [ $30 \mathrm{~nm} ; 12 \mathrm{~nm}$ ]. Numerical simulations on the electrical field intensity distributions show the absorption mechanisms for these structures that occur mainly in the TiN layers, either close to the surface at the wavelength of $700 \mathrm{~nm}$, or resonant in the whole volume at the wavelength of $500 \mathrm{~nm}$. The calculated emissivity in the 


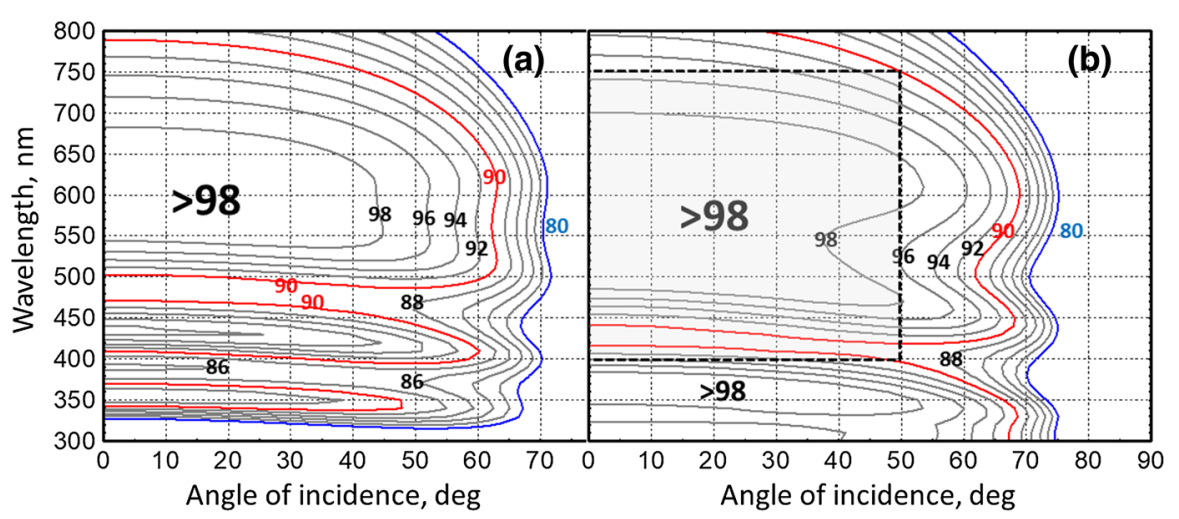

Fig. 9 Contour plot for the absorbance as a function of the angle of incidence ( $x$ axis) and as a function of the wavelengths ( $y$ axis): (a) $A_{a v}$ optimized structure [49 nm; $16 \mathrm{~nm}$ ] without silica; (b) $A_{a v}$ optimized structure $[30 \mathrm{~nm} ; 12 \mathrm{~nm}]$ with $\mathrm{d}_{\mathrm{SiO} 2}=50 \mathrm{~nm}$

Fig. 10 Maximum achievable absorbance $\mathrm{A}_{\mathrm{av}}$ as a function of the number of periods $N$. The histograms refer to optimized periodic multilayers with different materials: (black) ITO/TiN; (gold) ITO/Au; (gray) ITO/Ag (Color figure online)

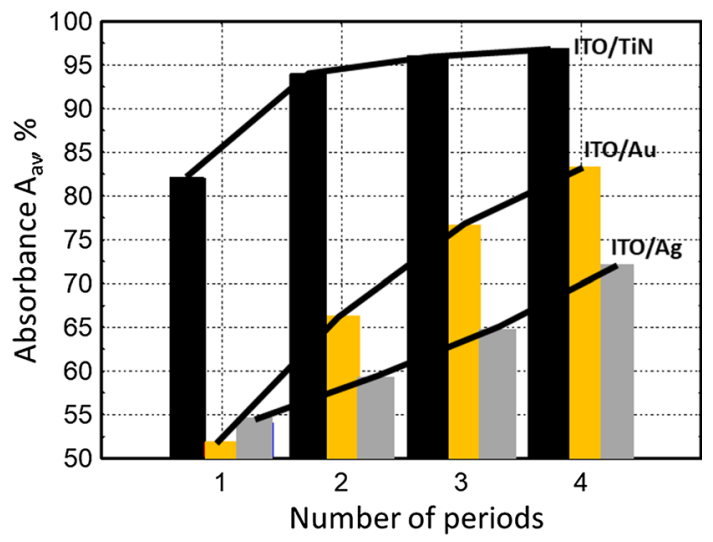

mid-infrared is always lower than $10 \%$, fulfilling the requirements even with a thin silica top layer.

To better understand the good quality of the results obtained with the ITO/TiN periodic multilayer we eventually made a comparison with other periodic multilayers by using noble metals such as, for example, ITO/Au (gold color) and ITO/Ag (gray color), in the same conditions. Figure 10 summarizes the results showing the maximum achievable $\mathrm{A}_{\mathrm{av}}$ when an increasing number of periods are used, for the different periodic multilayer structures. The highest absorbance is always obtained with ITO/TiN multilayers.

This confirms that the proposed perfect absorbers based on ITO/TiN periodic multilayer structures can achieve the best theoretical performance. In addition there are many other relevant advantages: the low cost of materials and fabrication process, the thermal stability, and the high melting temperature, which all make it a suitable coating material for solar collectors. 


\section{References}

1. Translated by F. Guthrie from Annalen der Physik: 109, 275-301 (1860): G. Kirchhoff (July 1860). "On the relation between the radiating and absorbing powers of different bodies for light and heat". The London, Edinburgh, and Dublin Philosophical Magazine and Journal of Science. Taylor \& Francis. 20 (130)

2. R. Siegel, J.R. Howell, Thermal Radiation Heat Transfer, vol. 1, 4th edn. (Taylor \& Francis, London, 2002), p. 7. ISBN 1-56032-839-8

3. Y. Nam, Y. Xiang Yeng, A. Lenert, P. Bermel, I. Celanovic, M. Soljačić, E.N. Wang, Solar thermophotovoltaic energy conversion systems with two-dimensional tantalum photonic crystal absorbers and emitters. Sol. Energy Mater. Sol. Cells 122, 287-296 (2014)

4. K. Mizuno et al., A black body absorber from vertically aligned single-walled carbon nanotubes. Proc. Natl. Acad. Sci. 106, 6044-6077 (2009)

5. Daniel D. Tune, B.S. Flavel, R. Krupke, J.G. Shapter, Carbon nanotube-silicon solar cells. Adv. Energy Mater. 2, 1043-1055 (2012)

6. G. Leahu, R. Li Voti, M.C. Larciprete, C. Sibilia, M. Bertolotti, I. Nefedov, I.V. Anoshkin, Int. J. Thermophys. 36, 1349-1357 (2015)

7. T. Cao, C.W. Wei, R.E. Simpson, L. Zhang, M.J. Cryan, Sci. Rep. 4, 3955 (2014)

8. W. Wang, S. Wu, K. Reinhardt, Y. Lu, S. Chen, Nano Lett. 10, 2012 (2010)

9. K. Aydin, V.E. Ferry, R.M. Briggs, H.A. Atwater, Nat. Commun. 2, 517 (2011)

10. T.V. Teperik, F.G. De Abajo, A. Borisov, M. Abdelsalam, P. Bartlett, Y. Sugawara, J. Baumberg, Nat. Photonics 2, 299 (2008)

11. C.W. Cheng, M.N. Abbas, C.W. Chiu, K.T. Lai, M.H. Shih, Y.C. Chang, Opt. Express 20, 10376 (2012)

12. M. Centini, A. Benedetti, M.C. Larciprete, A. Belardini, R. Li Voti, M. Bertolotti, C. Sibilia, Phys. Rev. B 92, 205411 (2015)

13. R. Li Voti, G. Leahu, M.C. Larciprete, C. Sibilia, M. Bertolotti, I. Nefedov, I.V. Anoshkin, Int. J. Thermophys. 36, 1342-1348 (2015)

14. A. Belardini, F. Pannone, G. Leahu, M.C. Larciprete, M. Centini, C. Sibilia, C. Martella, M. Giordano, D. Chiappe, F. Buatier De Mongeot, Appl. Phys. Lett. 100, 251109 (2012)

15. M. Laroche, R. Carminati, J.J. Greffet, Phys. Rev. Lett. 96, 123903 (2006)

16. I. Celanovic, D. Perreault, J. Kassakian, Phys. Rev. B 72, 075127 (2005)

17. C.G. Hu, L.Y. Liu, X.N. Chen, X.G. Luo, Opt. Express 17, 16745 (2009)

18. G. Leahu, R.L. Voti, C. Sibilia, M. Bertolotti, V. Golubev, D.A. Kurdyukov, Opt. Quantum Electron. 39, 305-310 (2007)

19. M.A. Kats, R. Blanchard, S. Zhang, P. Genevet, C. Ko, S. Ramanathan, F. Capasso, Phys. Rev. X 3, 041004 (2013)

20. Paonea, M. Geiger, R. Sanjines, A. Schüler, Solar Energy 110, 151-159 (2014)

21. G. Leahu, R. Li Voti, C. Sibilia, M. Bertolotti, Appl. Phys. Lett. 103, 231114 (2013)

22. R.L. Voti, M.C. Larciprete, G. Leahu, C. Sibilia, M. Bertolotti, J. Appl. Phys. 112, 034305 (2012)

23. Bingnan Wang, Thomas Koschny, Costas M. Soukoulis, Phys. Rev. B 80, 033108 (2009)

24. Eric Plum, Nikolay I. Zheludev, Chiral mirrors. Appl. Phys. Lett. 106, 221901 (2015)

25. A. Belardini, M. Centini, G. Leahu, D.C. Hooper, R. Li Voti, E. Fazio, J.W. Haus, C. Sibilia, Sci. Rep. 6, 31796 (2016)

26. A. Benedetti, B. Alam, M. Esposito, V. Tasco, G. Leahu, A. Belardini, R. Li Voti, A. Passaseo, C. Sibilia, Sci. Rep. 7, 5257 (2017)

27. J. Wu, C.Z. Zhou, H.C. Cao, A.D. Hu, Opt. Commun. 309, 57 (2013)

28. J.F. Zhou, H.T. Chen, T. Koschny, A.K. Azad, A.J. Taylor, C.M. Soukoulis, J.F. O’Hara, arXiv: 1111.0343

29. R. Li Voti, Rom. Rep. Phys. 64, 446-466 (2012)

30. M.C. Larciprete, A. Belardini, R.L. Voti, C. Sibilia, Opt. Express 21, A576-A584 (2013)

31. M.C. Larciprete, M. Centini, R.L. Voti, M. Bertolotti, C. Sibilia, Opt. Express 22, A1547 (2014)

32. A. Melnikov, A. Mandelis, J. Tolev, P. Chen, S. Huq, J. Appl. Phys. 107, 114513 (2010)

33. Q.M. Sun, A. Melnikov, A. Mandelis, Int. J. Thermophys. 37, 45 (2016)

34. O. Matsuda, M.C Larciprete, R. Li Voti, O.B. Wright, 56, Pages 3-20, (2015)

35. T. Dehoux, O.B. Wright, R.L. Voti, Ultrasonics 50, 197-201 (2010)

36. G.V. Naik, J. Kim, A. Boltasseva, Opt. Mater. Express 1, 1090 (2011) 
37. G.V. Naik, J.L. Schroeder, X. Ni, A.V. Kildishev, T.D. Sands, A. Boltasseva, Opt. Mater. Express 2, 478 (2012)

38. G.V. Naik, V.M. Shalaev, A. Boltasseva, Adv. Mater. 25, 3264 (2013)

39. J. Wang, C. Yin, M. Zhu, J. Sun, Kui Yi, Jianda Shao, Mod. Phys. Lett. B 31, 1750136 (2017)

40. N. Venugopal, V.S. Gerasimov, A.E. Ershov, S.V. Karpov, S.P. Polyutov, Opt. Mater. 72, 397-402 (2017)

41. Y. Hanjiang, T. Tan, W. Wei, C. Tian, Ying An, Fengjiu Sun, Current Appl. Phys. 12, 152-154 (2012)

42. Y. Liu, A. Mandelis, M. Choy, C. Wang, Lee Segal, Rev. Sci. Instrum. 76, 084902 (2005)

43. M. Abb, B. Sepu'lveda, M.H. Chong, O.L. Muskens, J. Opt. 14, 114007-1 (2012)

44. S. Rajak, M. Ray, J. Opt. 43, 231 (2014)

45. Chien-Cheng Kuo, Int. Sch. Sci. Res. Innov. 7, 570-572 (2013)

46. Jong-Bum You, Wook-Jae Lee, Dongshik Won, Yu. Kyoungsik, Opt. Express 22, 8339 (2014)

47. M. Scalora, M.J. Bloemer, A.S. Pethel, J.P. Dowling, C.M. Bowden, A.S. Manka, J. Appl. Phys. 83, 2377 (1998)

48. M.S. Sarto, R. Li Voti, F. Sarto, M.C. Larciprete, IEEE Trans. Electromagn. Compat. 47, 602-611 (2005)

49. R. Li Voti, M.C. Larciprete, G. Leahu, C. Sibilia, M. Bertolotti, J. Nanophoton. 6, 061601 (2012)

50. G. Cesarini, G. Leahu, M.L. Grilli, A. Sytchkova, Concita Sibilia, Roberto Li Voti, Phys. Status Solidi C 13, 998-1001 (2016)

51. J. Pflüger, J. Fink, W. Weber, K.P. Bohnen, G. Crecelius, Phys. Rev. B 30, 1155-1163 (1984)

52. M. Yuste, R. Escobar Galindo, O. Sánchez, D. Cano, R. Casasola, J.M. Albella, Thin Solid Films 518, 5720 (2010)

53. J.A. Briggs, G.V. Naik, Y.Zhao, T.A. Petach, Kunal Sahasrabuddhe, David Goldhaber Gordon, Nicholas A. Melosh, Jennifer A. Dionne, Appl. Phys. Lett. 110, 101901 (2017)

54. E.D. Palik, Handbook of Optical Constants of Solids (Academic Press, New York, 1985)

55. M. Born, E. Wolf, Principles of optics: electromagnetic theory of propagation, interference and diffraction of light (Pergamon Press, Oxford, 1964)

56. G. Leahu, E. Petronijevic, A. Belardini, M. Centini, R. Li Voti, T. Hakkarainen, E. Koivusalo, M. Guina, C. Sibilia, Sci. Rep. 7, 2833 (2017) 